\title{
No One Should Die of Melanoma: Time for This Vision to Be Realized?
}

\author{
Teresa Russo ${ }^{1}$, Aimilios Lallas ${ }^{2}$, Gabriella Brancaccio ${ }^{1}$, Vincenzo Piccolo ${ }^{1}$, \\ Roberto Alfano ${ }^{3}$, Giuseppe Argenziano ${ }^{1}$
}

\begin{abstract}
1 Dermatology Unit, University of Campania, Naples, Italy
2 First Department of Dermatology and Venereology, Aristotle University, Thessaloniki, Greece

3 Department of Anesthesiology, Surgery and Emergency, University of Campania, Naples, Italy
\end{abstract}

Citation: Russo T, Lallas A, Brancaccio G, Piccolo V, Alfano R, Argenziano G. No one should die of melanoma: Time for this vision to be realized? Dermatol Pract Concept. 2019;9(1):1-3. DOI: https://doi.org/10.5826/dpc.0901a01

Published: January 31, 2019

Copyright: $@ 2019$ Russo et al. This is an open-access article distributed under the terms of the Creative Commons Attribution License, which permits unrestricted use, distribution, and reproduction in any medium, provided the original author and source are credited.

Funding: None.

Competing interests: The authors have no conflicts of interest to disclose.

Authorship: All authors have contributed significantly to this publication.

Corresponding author: Teresa Russo, MD, II Policlinico, Edificio 9c, Primo piano, Via Pansini 5 - 80131 Naples, Italy. Email: russo. teresa87@gmail.com

In 1985, Ackerman said: "With big enough hopes and serious enough convictions, no human being need die of malignant melanoma" [1]. During the previous years, he had introduced the concept of "melanoma in situ" and proposed that melanoma can be histopathologically diagnosed when still confined within the epidermis. Based on this, he hypothesized that all melanomas could be diagnosed before invading the dermis and acquiring metastatic potential and, therefore, melanoma mortality would be eliminated.

It seems, however, that Ackerman was a bit ahead of his time. Even if melanoma could be histopathologically diagnosed before invading the dermis, how could such early melanomas be clinically recognized and excised? In that era, many melanomas were diagnosed only when ulcerating or bleeding. The ABCD clinical rule, which was introduced in the mid 80s, significantly helped clinicians to recognize melanoma earlier and patients to seek medical advice earlier. But even the ABCD rule is not usually sufficient to uncover melanoma in situ. This is because when the natural asymmetry of melanoma becomes visible to the naked eye, it is quite likely that the tumor has already invaded the dermis.

In the years that followed, a new diagnostic revolution occurred in the field of skin cancer: dermoscopy. The investigation of the sub-macroscopic morphology enabled the recognition of melanomas that did not have macroscopically detectable criteria. With the improvement of knowledge of dermoscopic morphology, thinner and thinner melanomas became recognizable.

Therefore, approximately 30 years after Ackerman expressed his vision, it seems that clinicians are now equipped with the necessary tools and knowledge to realize it. Today, melanoma can finally be recognized when still in situ or minimally invasive and, as such, people might stop dying from it. However, the harsh reality is that, although the incidence of thin melanoma has dramatically increased over the last decades, the incidence and mortality rates of thick melanoma seem to remain stable.

What are the main barriers to Ackerman's vision becoming reality? Whose fault is it that people still die of melanoma, and what can be done? 


\section{The tumor's fault!}

The melanoma family is comprised of tumors with different potentials to grow and metastasize. Fortunately, the great majority of melanomas grow superficially (slow-growing melanoma) for years before acquiring the biologic attitude of growing vertically (nodular component) and metastasizing [2]. However, rapidly growing melanomas do also exist (about $10 \%$ of melanomas diagnosed histopathologically) [2]. This type of biologically aggressive melanoma is largely responsible for melanoma mortality because it invades the dermis in only a few months, giving patients and doctors less of a chance to diagnose it at an early stage. Evidence suggests that nodular melanoma might grow vertically up to $0.5 \mathrm{~mm}$ per month [3]. This means that after only 3-4 months the lesion is already $1.5-2 \mathrm{~mm}$ in thickness, thus rapidly acquiring the potential to metastasize [4-6].

In fact, the problem of aggressive tumor biology might be even more difficult to solve, since it has been hypothesized that purely nodular melanoma might originate from dermal melanocytes. In 2008, Segura et al [7] investigated the morphologic features of melanomas with a nodular component and correlated in vivo reflectance confocal microscopy findings with histopathologic alterations. They found that, while superficial spreading melanomas with a nodular component displayed marked epidermal disarrangement and pagetoid infiltration, purely nodular melanomas showed a preserved epidermis with only a few pagetoid cells. These observations, along with the report of "primarily dermal melanomas" a few years earlier revisited the conventional concept that melanoma develops only from transformed epidermal melanocytes, proposing instead that some melanomas might originate from dermal stem cells.

The problem of "purely nodular," "primary dermal," or rapidly growing melanoma becomes even more complicated in the light of evidence suggesting that these melanomas develop in patients with a low nevus count and without other known risk factors. Given that most screening strategies are designed on the basis of known risk factors, it seems that they fail to target individuals that will develop fast-growing melanomas.

Although purely nodular tumors represent a small proportion of all melanomas, they seem to have a definite impact on melanoma mortality. Eliminating this impact is probably impossible, especially if it is true that some of these tumors originate in the dermis. Reducing the impact could be possible by reviewing the screening strategies so as to better target individuals at risk and by promoting self-examination. The rapidly developing artificial intelligence diagnostic software could potentially help to improve and expand populationbased screening.

\section{The patient's fault!}

In everyday practice, many melanomas are very easy to recognize clinically. To a certain extent, this is because they are so advanced and display all the typical morphologic characteristics of melanoma. Many patients presenting with advanced melanomas report not to have sought medical advice earlier even if the tumor had been present for several months or even years. This thoughtless behavior can be attributed to a lack of awareness and to the widespread and popular misconceptions. Characteristically, a survey among 1,024 adult Italian women showed that $82 \%$ believed that excising a mole is dangerous because the surgical procedure could turn a benign mole into a malignant one [8]. Another survey performed in the US reported that approximately $20 \%$ of the participants "always thought that melanoma was not very serious."

An association between the socio-economic status and melanoma thickness has been reported in several populationbased studies. For example, Pollitt et al studied 566 patients with newly diagnosed melanoma and confirmed that a low socioeconomic status was associated with increased melanoma thickness and decreased survival [9]. In 2008, Schneider et al showed that an employee education and screening program at Lawrence Livermore National Laboratory resulted in a significant reduction of melanoma mortality [10].

Screening programs are considered the main solution to increased public awareness. Indeed, widely applied screening strategies for breast or prostate cancer seem to have succeeded in increasing awareness, even though it is not always clear if they also succeed in reducing mortality. For skin cancer, screening programs are not so well established, but they do exist. An example was the SCREEN project, during which a population-based skin cancer screening was performed in one state in Germany. Then, melanoma incidence was compared to that in another state, where the screening was not performed. As expected, the investigators found an increased melanoma incidence in the state where the screening took place. The major differences between the 2 states occurred among women, the group with the greatest SCREEN participation. These results are consistent with the impact of effective screening for other cancers [11,12].

However, screening programs for skin cancer might not be equally effective in increasing awareness if they are not combined with a systematic effort to dispel widespread misconceptions. We need to realize that a significant proportion of patients with advanced melanoma delay seeking medical attention, not because they do not see their melanomas, but because they think that it is not dangerous or, even worse, that it would be dangerous to excise it.

Therefore, a dual effort is required to improve public behavior concerning melanoma detection. First, improved and widespread screening programs should be available and 
easily accessible. Screening will allow clinicians to recognize early melanomas that patients themselves cannot detect. Second, the population should be better educated to perform self-examination and, mainly, to immediately seek medical advice for melanomas that they do detect but neglect because of misconceptions.

\section{The doctor's fault!}

The likely reason why doctors miss melanoma is not their inability to recognize it, but the fact that they do not give themselves the chance to see and examine it. It has been shown that a big proportion of melanomas are "diagnosed" by the patients themselves, meaning that the patients seek medical advice precisely because they are worried about a lesion that finally proves to be melanoma. The remaining melanomas, however, remain to be discovered by clinicians. To do so, clinicians have to examine clinically and dermoscopically all the patient's moles. They do so when patients ask for it, but when patients come in for other focused symptoms, evidence suggests that clinicians usually do not offer a total body mole check. It has been reported that up to $63 \%$ of patients diagnosed with melanoma had visited their general practitioner within the year prior to the diagnosis for another medical problem. Dermatologists do not do much better, with only $30 \%$ of them performing a total body skin examination on all their patients.

Although this is understandable within the frame of a busy daily practice, clinicians should take into consideration data suggesting that 1 skin cancer is found every 50 patients examined with total body skin check, and 1 melanoma is uncovered every 400 patients. Reading these data inversely, a clinician would realize that with every 50 patients he/she examines without a total body check, 1 skin cancer is missed, and with every 400 patients 1 melanoma is overlooked.

Although the safest proposal would be to offer a totalbody skin examination to all patients seeking dermatologic consultations, it might be unrealistic to apply in the real practice. However, a total-body skin check should be offered at least to individuals belonging to high-risk groups [13-15].

The epidemiologic evidence is well known: high-risk groups include individuals with (1) personal history of skin cancer; (2) more than 20 nevi on the arms, which is predictive of a high total nevus count; and (3) sun-damaged skin on uncovered areas. The strategy of total-body skin checks would be fruitful if applied not only by dermatologists, but also by appropriately trained general practitioners (GPs). Grange et al [16] found that training GPs on skin cancer diagnosis and encouraging them to perform systematic skin examinations might reduce the mean Breslow thickness of diagnosed melanomas and the incidence of very thick melanomas.

\section{Conclusions}

We strongly believe that people still die of melanoma for reasons that are related to culture, general education, and habits of doctors and patients rather than reasons related to the lack of scientific knowledge or insufficiency of diagnostic tools. Realizing this might help the medical community and health authorities to better target their efforts.

\section{References}

1. Ackerman AB. No one should die of malignant melanoma. J Am Acad Dermatol. 1985;12(1 Pt 1):115-116.

2. Greenwald HS, Friedman EB, Osman I. Superficial spreading and nodular melanoma are distinct biological entities: a challenge to the linear progression model. Melanoma Res. 2012;22(1):1-8.

3. Liu W, Dowling JP, Murray WK, et al. Rate of growth in melanomas: characteristics and associations of rapidly growing melanomas. Arch Dermatol. 2006;142(12):1551-1558.

4. Brancaccio G, Russo T, Lallas A, Moscarella E, Agozzino M, Argenziano G. Melanoma: clinical and dermoscopic diagnosis. G Ital Dermatol Venereol. 2017;152(3):213-223.

5. Russo T, Piccolo V, Lallas A, et al. Dermoscopy of malignant skin tumours: what's new? Dermatology. 2017;233(1):64-73.

6. Russo T, Piccolo V, Ferrara G, et al. Dermoscopy pathology correlation in melanoma. J Dermatol. 2017;44(5):507-514.

7. Segura S, Pellacani G, Puig S, et al. In vivo microscopic features of nodular melanomas: dermoscopy, confocal microscopy, and histopathologic correlates. Arch Dermatol. 2008;144(10):1311-1320.

8. Piccolo V, Russo T, Giacomel J, Lallas A, Alfano R, Argenziano G. Dispelling myths concerning pigmented skin lesions. J Eur Acad Dermatol Venereol. 2016;30(6):919-925.

9. Pollitt RA, Swetter SM, Johnson TM, Patil P, Geller AC. Examining the pathways linking lower socioeconomic status and advanced melanoma. Cancer. 2012;118(16):4004-4013.

10. Schneider JS, Moore DH 2nd, Mendelsohn ML. Screening program reduced melanoma mortality at the Lawrence Livermore National Laboratory, 1984 to 1996. J Am Acad Dermatol. 2008;58(5):741-749.

11. Swetter SM, Pollitt RA, Johnson TM, Brooks DR, Geller AC. Behavioral determinants of successful early melanoma detection: role of self and physician skin examination. Cancer. 2012;118(15):3725-3734.

12. Waldmann A, Nolte S, Weinstock MA, et al. Skin cancer screening participation and impact on melanoma incidence in Germany-an observational study on incidence trends in regions with and without population-based screening. Br J Cancer. 2012;106(5):970974.

13. Argenziano G, Zalaudek I, Hofmann-Wellenhof R, et al. Total body skin examination for skin cancer screening in patients with focused symptoms. J Am Acad Dermatol. 2012;66(2):212-219.

14. Hoorens I, Bossaert K, Pil L, et al. Total-body examination vs lesion-directed skin cancer screening. JAMA Dermatol. 2016;152(1):27-34.

15. Federman DG, Kravetz JD, Kirsner RS. Skin cancer screening by dermatologists: prevalence and barriers. J Am Acad Dermatol. 2002;46(5):710-714.

16. Grange F, Woronoff AS, Bera R, et al. Efficacy of a general practitioner training campaign for early detection of melanoma in France. Br J Dermatol. 2014;170(1):123-129. 